\title{
Introducing Palliative Care within the Treatment of End-Stage Liver Disease: The Study Protocol of a Cluster Randomized Controlled Trial
}

\author{
Manisha Verma, MD, MPH, ${ }^{1}$ Andrzej S. Kosinski, PhD, ${ }^{2}$ Michael L. Volk, MD, ${ }^{3}$ \\ Tamar Taddei, MD, ${ }^{4}$ Kavitha Ramchandran, MD, ${ }^{5}$ Marie Bakitas, DNSc, NP-C, FAAN, ${ }^{6}$ \\ Kenneth Green, ${ }^{7}$ Lisa Green, ${ }^{7}$ and Victor Navarro, MD ${ }^{1}$
}

\begin{abstract}
Introduction: Patients with end-stage liver disease (ESLD) suffer from myriad symptoms due to the systemic effects of the disease and unpredictable acute episodes, which contribute to progressive deterioration in quality of life (QOL). Despite clear evidence that palliative care (PC) improves QOL in other serious illnesses, PC is underutilized and delayed for ESLD patients. Through a comparative effectiveness trial of specialist led consultative PC (Model 1) versus trained hepatologist led PC (Model 2), we aim to build evidence on introducing
\end{abstract} PC into the routine outpatient care of ESLD patients.

Objective: We hypothesize that trained hepatologist led PC model will have a better improvement in QOL compared to consultative PC model.

Methods: This two-arm, multicenter cluster-randomized trial assesses the effectiveness of two PC models for patients with ESLD. Fourteen clinical centers will recruit 1260 patient-caregiver dyads. Each center is the unit of randomization. Hepatologists at sites randomized to the Model 2 have undergone web-based training in the principles of PC as pertained to ESLD. PC intervention is delivered over four visits (initial, one, two, and three months). Follow-up assessments occur at 6, 9, and 12 months. Eligible patients are those with new onset or ongoing complications of ESLD with a caregiver willing to participate.

Outcomes: The primary outcome is change in patients' QOL from baseline to three months. Secondary outcomes include symptom burden, depression, distress, satisfaction with care, caregiver burden and QOL, goal concordant care, and health care utilization.

Challenges and Contributions Engagement: A research advisory board has been developed with representatives from the participating centers, who have provided active feedback on the protocol, outcomes, study methods, and training program.

Intervention Fidelity: Intervention fidelity will be maintained by adherence to a visit agenda and providers in both models completing a PC checklist after each study visit.

Keywords: end-stage liver disease; hepatology and palliative care; models of health care delivery

Introduction

$\mathbf{E}$ ND-STAGE LIVER DISEASE (ESLD) is 1 of the 10 leading causes of death in United States ${ }^{1}$ and the 7 th leading cause of death in persons aged 25-64 years. ${ }^{2}$ It is commonly associated with high symptom burden, frequent hospitaliza- tions, and mortality similar to those with cancer. ${ }^{3-5}$ These factors are linked with significant deterioration in quality of life (QOL) and immense caregiver burden. ${ }^{6}$ Liver Transplantation (LT), the only curative treatment, is available only to a minority of those affected given the scarcity of donor organs. In addition, the fluctuating trajectory of ESLD

\footnotetext{
${ }^{1}$ Department of Digestive Diseases and Transplantation, Einstein Healthcare Network, Philadelphia, Pennsylvania.

${ }^{2}$ Department of Biostatistics, Duke Clinical Research Institute, Durham, North Carolina.

${ }^{3}$ Department of Gastroenterology and Hepatology, Loma Linda University, Loma Linda, California.

${ }^{4}$ Department of Digestive Diseases, Yale School of Medicine, New Haven, Connecticut.

${ }^{5}$ Division of Medical Oncology, Stanford University, Stanford, California.

${ }^{6}$ Center for Palliative and Support Care, The University of Alabama at Birmingham, Birmingham, Alabama.

${ }^{7}$ Research Advisory Board, Einstein Healthcare Network, Philadelphia, Pennsylvania.

Accepted April 30, 2019
} 
associated with sudden decompensating events makes the prognosis uncertain. ${ }^{7}$ ESLD patients and caregivers are left with unpredicted hope and suffering. ${ }^{8}$ Hence there is an urgent need for effective interventions to improve QOL and enhance the overall care for ESLD. ${ }^{9}$

Palliative care (PC) is a relatively new medical specialty that provides an extra layer of support to usual medical care, improving QOL and symptoms by addressing the holistic needs of patients and their caregivers. ${ }^{10} \mathrm{PC}$ has shown to reduce health care costs, improve the quality of care, and align the goals of care between patients/caregivers and their providers for several serious illnesses. ${ }^{11-13}$ Existing research demonstrates underutilization of PC for liver disease. ${ }^{14} \mathrm{PC}$ is stigmatized and remains conflated with hospice and "giving up." ${ }^{15}$ With the increasing PC demands, but limited number of PC training programs, there exists a shortage of formally trained PC providers. This leads to limited availability of outpatient PC service. In addition, the hepatologists have limited knowledge of how to provide PC. These aspects create a critical gap in introducing PC within routine care of ESLD patients and their caregivers. ${ }^{16,17}$ We aim to fill this gap by introducing PC within routine hepatology care, through comparative clinical effectiveness research of two health care delivery models.

Research shows that non PC providers can be trained to offer PC. ${ }^{18}$ Universal training of all health care providers in $\mathrm{PC}$ is considered a top priority of patient-centered care paradigm. ${ }^{19}$ There is an argument that the hepatologists must be the chamberlains of PC for ESLD. ${ }^{20}$ Hence we propose to establish a hepatologist-led PC model involving a formal PC training followed by delivery of PC in outpatient hepatology practice and juxtapose it to a specialist led consultative $\mathrm{PC}$, in which patients will be referred to see PC providers, who then render their service as a part of their standard practice.

We set forth a pragmatic study to compare and contrast:

Model 1: Specialist led consultative PC (i.e., PC offered by board-certified or board-eligible PC provider), versus.

Model 2: Trained hepatologist-led PC (i.e., after formal training, the hepatologist delivers PC services integrated into the liver clinic).

\section{Objectives and Hypotheses}

Our primary objective is to support introduction of PC for ESLD population, through comparing two health care delivery models.

\section{Primary hypothesis}

Our primary hypothesis is that patients receiving $\mathrm{PC}$ through the hepatologist-led model (Model 2) will demonstrate a greater improvement in QOL (as measured by FACT-Hep or functional assessment of cancer therapyhepatobiliary) from baseline to three months compared to the consultative PC model (Model 1). The scientific premise of the primary hypothesis is that non PC providers can be trained to offer PC and that hepatologist-delivered PC will build upon an existing therapeutic relationship and expert knowledge of liver disease, prognosis, and disease trajectory, allowing them to better individualize and integrate PC into routine hepatology care.

\section{Secondary hypotheses}

1. We hypothesize that both PC models will lead to improved symptoms, depression, and satisfaction with care, from baseline to three months, and that Model 2 (hepatologist led) will have superior improvements than Model 1 (consultative PC).

2. We hypothesize that the PC interventions in both Models will improve patient distress, caregiver burden, and QOL from baseline to three months, with better improvement in Model 2.

3. We also hypothesize that both models will lead to reduced health care utilization from baseline (assessed using 30- and 90-day admissions, emergency room (ER) visits, and unscheduled office visits).

\section{Exploratory hypothesis}

We hypothesize that the improvement in QOL will be sustained at 6,9 , and 12 months and that both models will have prolonged survival compared to historical data.

\section{Methods}

\section{Study overview}

This is a multicenter, cluster-randomized comparative effectiveness study of two pragmatic health care delivery models of PC for patients with ESLD, that is, consultative PC (Model 1) versus trained hepatologist-led PC (Model 2) (Fig. 1).

Fourteen clinical centers acted as the clusters and are randomized to either of the two models. All the patient/caregiver dyads within a cluster will receive the same intervention. Clinical centers are randomized within two strata as follows: Veterans Administration (VA) centers and non-VA academic medical centers. Each enrolled patient/caregiver dyad will participate for 3 months of study intervention and 9 months of follow-up for a total of 12 months of participation.

Parallel to this cluster-randomized controlled trial, a qualitative study will be undertaken to evaluate the patient/caregiver and health care providers,' experiences in the two PC models using semistructured interviews. We will also assess hepatologists' perception of knowledge and skill acquisition and confidence with symptom management in Model 2.

\section{Setting}

Seven VA and seven non-VA clinical centers were recruited through consortia of hepatologists who are members of the American Association for the Study of Liver Diseases (Fig. 2).

\section{Study population}

Patients with ESLD who have a recent episode of decompensation and/or patients with hepatocellular cancer (with a prognosis of at least three months) with a caregiver willing to participate will be eligible. A caregiver is defined as someone who knows the patient well and is involved in their routine medical care.

Table 1 outlines the detailed inclusion/exclusion criteria.

\section{Study intervention}

The intervention in this study involves referring patients to PC providers (board certified or eligible) at their respective 


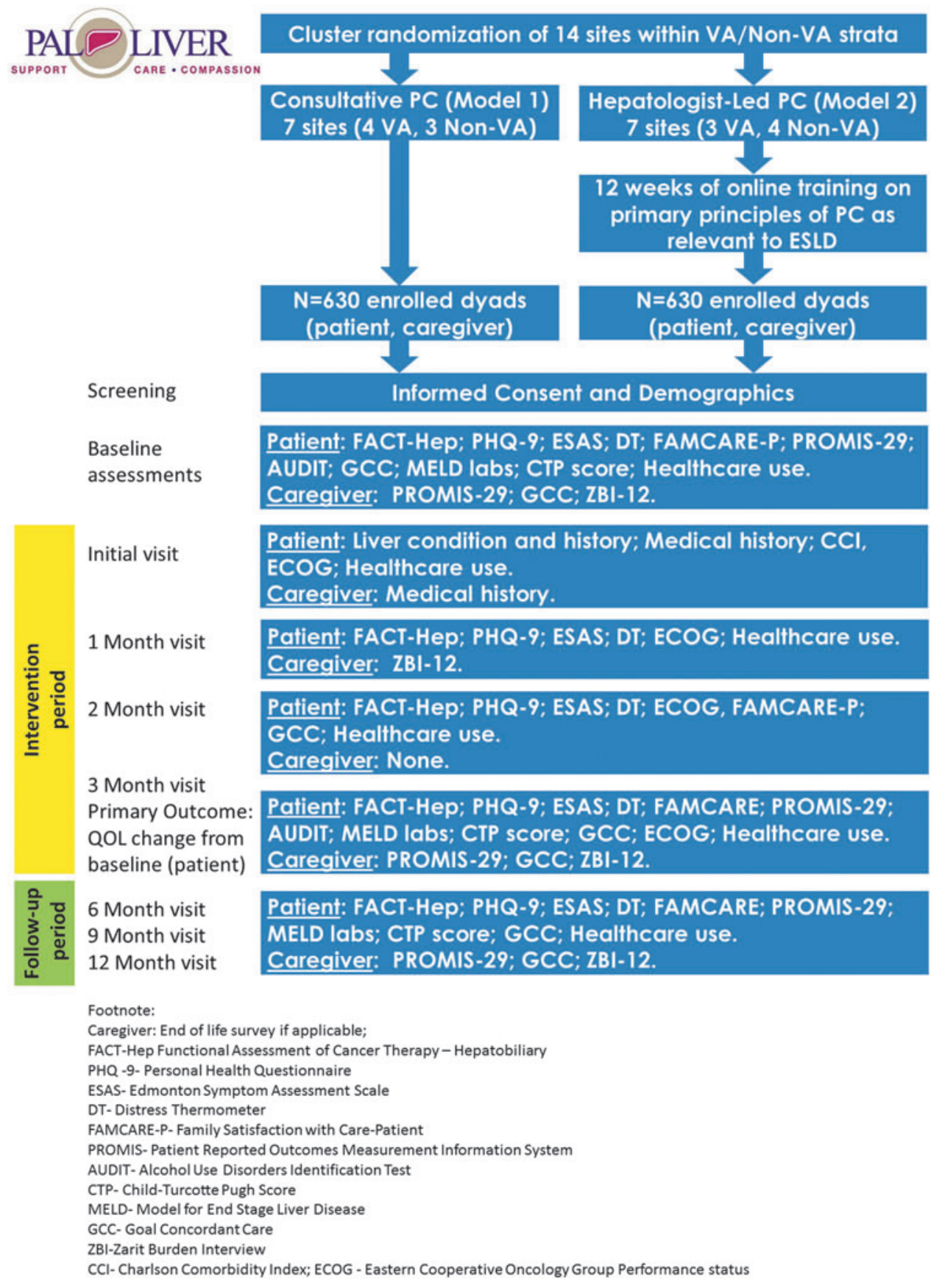

FIG. 1. Study design. ESLD, end-stage liver disease; PC, palliative care; VA, Veterans Administration.

institutions in Model 1, who then render PC as their standard service, and PC delivered by hepatologists (who have undergone a formal PC training) within routine hepatology practice in Model 2. The hepatologist training program is described below.

All consented dyads in both models receive PC service at the initial visit and at one, two, and three months from the initial visit. Providers in both models will use a PC checklist (Table 2) to assure delivery of key elements of PC. This checklist is based on American Society of Clinical Oncology (ASCO) Ambulatory Palliative Care Guidelines. ${ }^{21}$ The key elements include the following: patients'/caregivers' under- standing of diagnosis, illness and prognosis, symptom assessment, psychosocial assessment, distress screening and management, discussion of goals of care, and advanced directives.

In general, the providers in both models will conduct clinical assessment at each visit and offer a recommendation for symptoms, depression, and distress; discuss goals of care; and help with treatment decision making. The study visits are not scripted in either of the models, allowing the providers freedom to address individual patient needs and support the pragmatic nature of this trial. Both models will bill for their medical service, as a part of routine clinical care. 


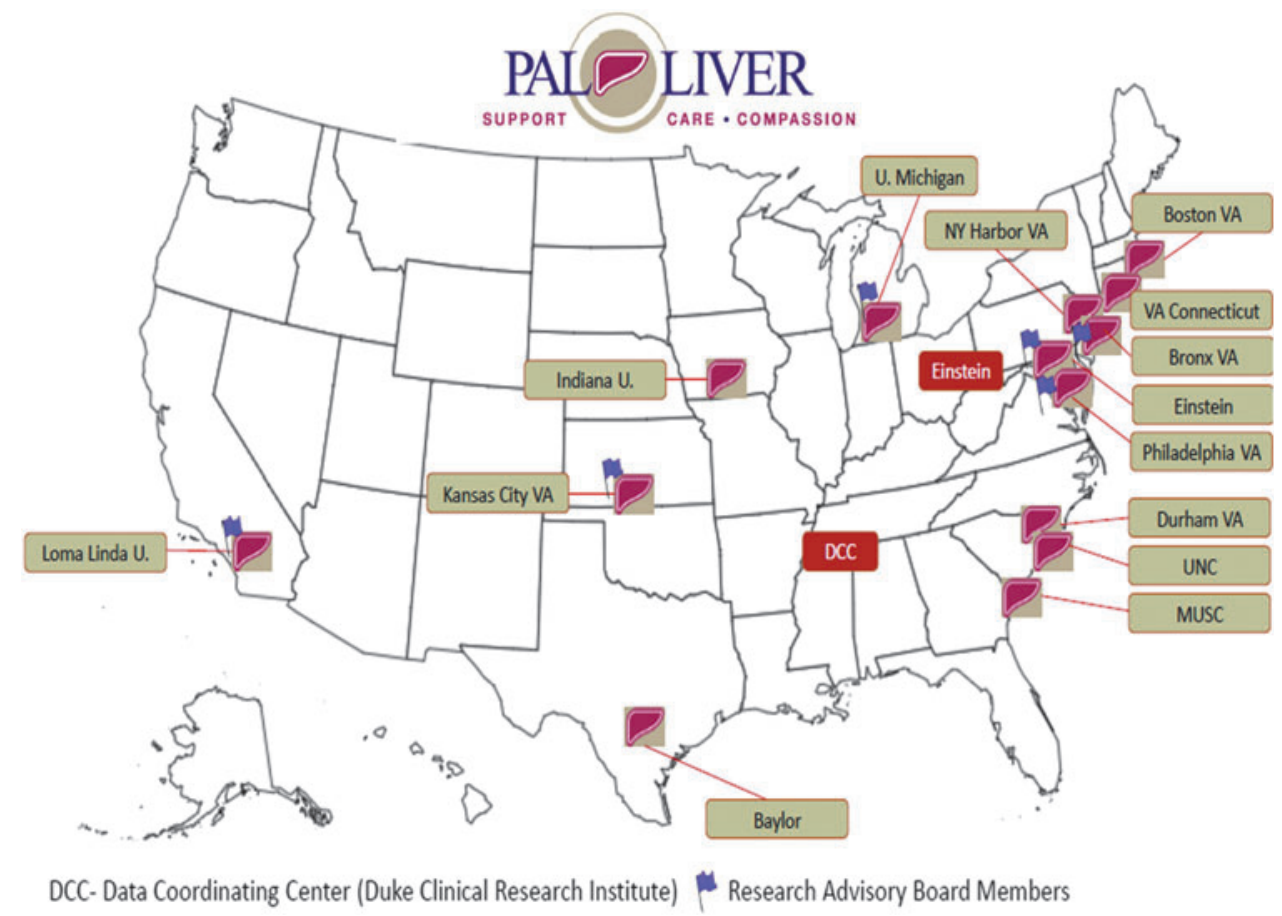

FIG. 2. PAL-LIVER Clinical Network. MUSC, Medical University of South Carolina; UNC, University of North Carolina.

\section{Palliative care always-hepatology training program}

We partnered with Stanford online learning experts to design a palliative care always-hepatology (PCA-Hep) course (Fig. 3). The course was designed based on specific scenarios of ESLD, such as transplant eligible versus ineligible, delisting from transplant list, acute episodes of decompensation, and uncertain prognosis. The course aims to teach primary PC skills to hepatologists.

There are 11 modules, and each module includes objectives, content directed to clinical practice, vignettes outlining how to assess and address the common needs of ESLD

\section{Palliative \\ Care Always: \\ Hepatology}

\section{Palliative Care Always: Hepatology}

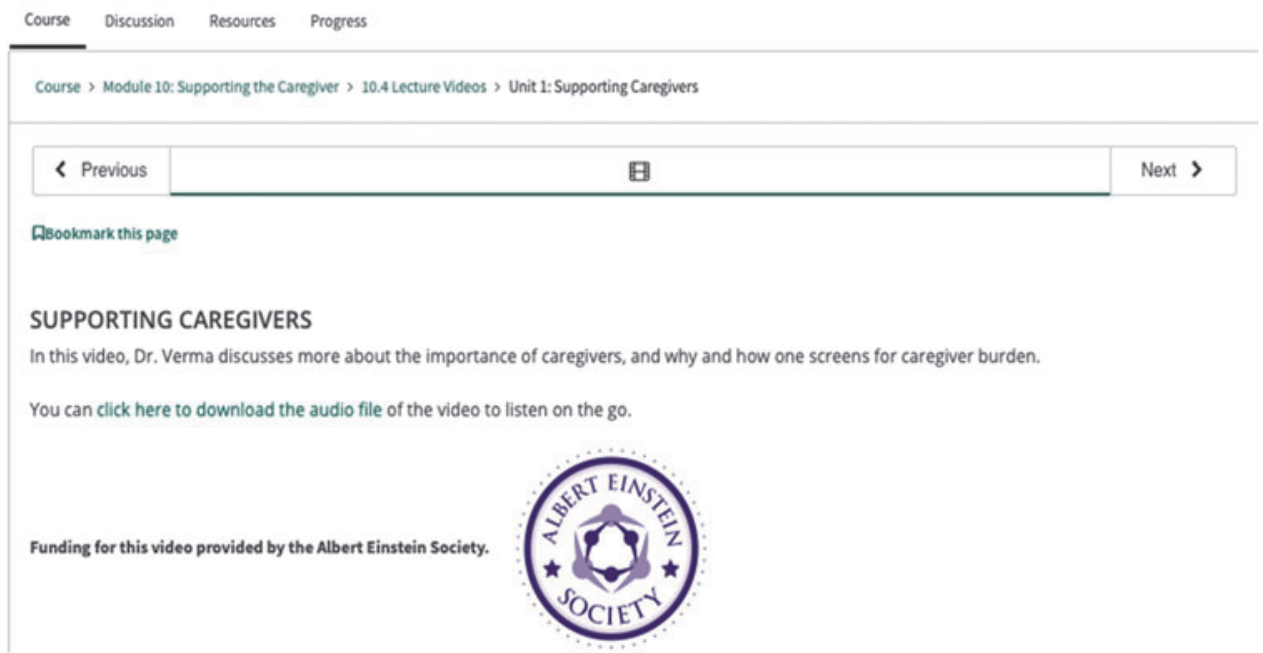

FIG. 3. Snapshot of palliative care always-hepatology training program. 
TABle 1. INCLUSION AND EXClusion CRITERIA

Inclusion criteria: patients

1) Age $>18$ years old

2) Patient and caregiver willing to sign informed consent

3) Able to read/understand English

4) Either of the following two clinical criteria:

I. CTP Class B or C cirrhosis with one of the following (new or ongoing) within the prior six months from the date of consent:

1. Ascites (requiring diuretics)

2. Spontaneous bacterial peritonitis

3. Hepatic hydrothorax (requiring diuretics)

4. Variceal bleed (with one or more recurrences)

5. Overt hepatic encephalopathy (requiring medications)

6 . Type 2 hepatorenal syndrome

II. Hepatocellular cancer:

1. Any BCLC (except Stage D) with CTP class B OR

2. BCLC Stage $C$ with CTP class A

Exclusion criteria: patients

1) Hepatologist-estimated life expectancy of less than six months

2) Prior LT

3) MELD $>30$ at the time of enrollment

4) Patients who, in the judgment of the investigator, are likely to undergo LT within three months of enrollment

5) Lacks capacity to provide informed consent, including those with stage 2 hepatic encephalopathy (HE) or higher at the time of consent

6) Patients who are already receiving or who have received palliative care before study entry (within the past 1 year)

Inclusion criteria: caregivers

1. Identified caregiver of ESLD patients

2. Age $>18$

3. Able to read/understand English

4. Providing direct care for at least $>10$ hours per week

Exclusion criteria: caregivers

1. Impaired cognitive function

BCLC, Barcelona Clinic Liver Cancer; CTP, Child-Turcotte-Pugh; LT, liver transplantation; MELD, model for end-stage liver disease.

patients and their caregivers, and additional PC resources (Table 3). The learning approach includes readings and videos, coupled with pre- and postmodule assessment questions. The content is based on existing evidence-based literature and from conventional PC practice. A certificate is awarded with a minimum passing score of $80 \%$ after each module. The training occurred over a 12 -week period, requiring approximately 3-5 hours per week. Twenty-five hepatologists from seven clinical centers in Model 2 completed the training successfully. The course was moderated by the course director (K.R.) and her team, and web-based live sessions were conducted every four weeks. During the live webinars, providers were able to practice the skills learned from each module. An online refresher will be required every six months during the recruitment period to maintain the skills learned.

Below are details for each module:

Module 1: Introduction to $P C$ is based on the National Consensus Guidelines for $\mathrm{PC}^{22}$ and the Institute of Medicine's report on improving quality and honoring individual preferences near the end of life. ${ }^{23}$

Module 2: Communication with Patients and Families is based on widely used OncoTalk training ${ }^{24}$ and the six steps of SPIKES criteria (Setting, Perception, In- formation, Knowledge, Empathy, and Summarize) to approach breaking bad news. ${ }^{25}$ Emphasis is placed on talking about prognosis, conducting a family meeting, and advance care planning.

Module 3: Psychosocial Support is taught through an introduction to screening for psychosocial distress (using distress thermometer [DT] $)^{26}$ and distress management based on the National Comprehensive Cancer Network clinical practice guidelines. ${ }^{27,28}$

Module 4: Goals of Care module outlines: (1) assessing ongoing physical, emotional, and practical needs and (2) documentation of preferences in case of terminal illness. This is based on REMAP tool (Reframe, Expect emotion, Map outpatient goals, Align with goals, and Propose a plan). ${ }^{29}$ The Name, Understand, Respect, Support, and Explore approach is promoted to be used to identify and respond to emotional cues during goals of care development. ${ }^{30}$ In addition, five steps to advance care planning are discussed (introduce the topic, engage in structured discussions, document patient preferences, review and update the directive, and apply prior directives to actual circumstances). ${ }^{31}$

Module 5: Symptom Management: Physical Symptoms. This module outlines the symptoms commonly associated 
Table 2. Study Intervention: Palliative Care Checklist

Elements to be assessed

Visit

Visit
No. 1

One

Two

Three

Patient

Assessment

1. Assess patient understanding of diagnosis, illness, and prognosis

2. Conduct distress screening (DT) and evaluate current management

3. Conduct symptom assessment (ESAS) and determine management plan

4. Conduct a psychosocial assessment and evaluate management

5. Conduct depression screening (using PHQ-9) and determine current or future need for management plan

6. Assess social role (occupation and social role) and family support

7. Assess spiritual, religious, and cultural beliefs that impact illness

8. Identify changes in liver conditions that trigger need for treatment decision making and shifting goals

9. Discuss and document goals of care

10. Conduct ACP (assessment and presence of proxy decision maker, completion of advanced directives) (CAN OCCUR AT ANY VISIT)

Planning/recommendations

1. Identify care plan for future appointments

2. Document new prescriptions or change in dose of existing meds

3. Referrals: social work, palliative care, nutrition, physical/occupational therapy, etc.

Caregiver

1. Assess caregiver understanding of diagnosis, illness, and prognosis

2. Validate the normal feelings of stress, etc associated with caregiving

3. Assess caregiver coping and material support resources

4. Assess caregiver burden and document concerns

5. Refer for support resource to alleviate caregiver burden and/or document ways to relieve the burden and self-care

6. Assess for educational needs related to patient's condition: symptom triggers for doctor's attention, nutritional needs, understanding of complications, and ACP

7. Discuss future care management and treatment options

$\begin{array}{llll}\text { REQ } & \text { REQ } & \text { REQ } & \text { REQ } \\ \text { REQ } & \text { REQ } & \text { REQ } & \text { REQ } \\ \text { REQ } & \text { REQ } & \text { REQ } & \text { REQ } \\ \text { REQ } & \text { prn } & \text { prn } & \text { prn } \\ \text { REQ } & \text { prn } & \text { prn } & \text { prn } \\ & & & \\ \text { REQ }^{\text {a }} & & \text { prn } & \text { prn } \\ \text { REQ }^{\text {a }} & & \text { prn } & \text { prn } \\ \text { prn }^{2} & \text { prn } & \text { REQ } & \text { prn } \\ \text { REQ }^{\text {a }} & & & \\ \text { REQ }^{\mathrm{a}} & & \text { prn } & \text { prn } \\ & & \text { prn } & \text { prn } \\ & & & \\ \text { REQ } & \text { REQ } & \text { REQ } & \text { REQ } \\ \text { REQ } & \text { prn } & \text { prn } & \text { prn } \\ \text { REQ } & \text { prn } & \text { prn } & \text { prn }\end{array}$

$\begin{array}{llll}\text { REQ } & \text { prn } & \text { prn } & \text { prn } \\ \text { REQ }^{\mathrm{a}} & & \text { prn } & \text { prn } \\ & \text { REQ } & \text { prn } & \text { prn } \\ \text { REQ } & \text { prn } & \text { prn } & \text { prn } \\ \text { REQ } & \text { prn } & \text { prn } & \text { prn } \\ & \text { REQ } & \text { prn } & \text { prn }\end{array}$

${ }^{a} \mathrm{REQ}=$ must be conducted within the first two visits.

ACP, advance care planning; ESAS, Edmonton Symptom Assessment Scale; PHQ-9, Patient Health Questionnaire; prn, as needed; REQ, required.

with ESLD and their treatments. A method to conduct basic symptom and pain assessment using Edmonton Symptom Assessment Scale (ESAS) is highlighted.

Module 6: Symptom Management: Psychological Symptoms. This module outlines common causes of emotional distress related to liver disease, the difference between appropriate sadness and depression, and utilizing patient's thoughts, feelings, and bodily reactions to screen and assess emotional distress. Formal de-

\section{Table 3. Palliative Care Always-Hepatology} TRAINING PROGRAM

\footnotetext{
Module 1: Introduction to palliative care and the PALLIVER study

Module 2: Communicating with patients and families

Module 3: Psychosocial support

Module 4: Discussing goals of care

Module 5: Symptom management: physical symptoms

Module 6: Symptom management: psychological symptoms

Module 7: Spiritual care

Module 8: Hospice

Module 9: Survivorship

Module 10: Caregiver support

Module 11: Epilogue (summary of course and billing guidelines)
}

pression screening using Patient Health Questionnaire (PHQ-9) is suggested.

Module 7: Spiritual Care training is based on the guidelines of the National Consensus Project Guidelines and the National Quality Forum Preferred Practices. ${ }^{32}$ It outlines several expressions of spirituality, the benefits of spiritual screening and assessment, screening for spiritual distress, the role of a chaplain in hepatology care, how hope and forgiveness serve a role in the life of a seriously ill patient, and identify specific steps that can be taken to cultivate hope and forgiveness.

Module 8: Hospice. This module defines hospice care and how it differs from PC. It identifies strategies to help patients and families understand the benefits of hospice and to describe how a patient's needs change as the prognosis changes.

Module 9: Survivorship. This module describes paths of survivorship care for the various types of survivors of ESLD, for example, Post liver transplant. The Cancer Survivor Toolbox (from the National Cancer Coalition) is utilized as the resource and tailored to liver disease specific needs. ${ }^{33}$ In addition, the ASCO Survivorship care plan is outlined.

Module 10: Caregiver Support is based on methods to assess and manage caregiver burden. We discuss the 
emotional toll of caregiving for patients with ESLD and delineate common stressors for caregivers.

Module 11: Epilogue is a summary of all the modules described above and adds guidance on additional revenue/billing for PC services.

\section{Study Outcomes \\ Primary outcome}

Patient reported QOL (assessed using FACT-Hep total score) is the primary measure of this study. The primary outcome will be the change in QOL from baseline to the QOL assessment at three months. Further assessments will be conducted at 6,9 , and 12 months to assess the sustainability effects.

\section{Secondary outcomes}

The outcomes inset below will be assessed as a change from baseline to three months:

- Overall QOL (PROMIS-29 or Patient Reported Outcomes Measurement Information System)

- Symptom burden (modified ESAS)

- Depression severity (PHQ-9)

- Distress (DT)

- Satisfaction with care (Family Satisfaction with Cancer Care-Patient scale)

- Caregiver burden (Zarit Burden Interview-12) and caregiver QOL (PROMIS-29)

- Goal concordant care (patient and caregiver questionnaire)

Health care utilization:

- Rate of hospital admissions within 30 and 90 days of enrollment

- Rate of ER visits within 1 year of enrollment

- Rate of unscheduled office visits within 1 year of enrollment.

\section{Exploratory outcome}

Survival.

\section{Qualitative outcomes}

Thematic qualitative description of patient, caregiver, and providers' experiences with the two models. In addition, knowledge gained by the hepatologists from the PC training program will be described.

\section{End-of-life care}

The caregivers will be approached to complete the Kaiser Permanente end-of-life survey in the event of patient death during the time of their participation. These questions will be completed within three months of death.

\section{Study procedures}

Patients will be identified and consented at the time of their routine hepatology clinical visit. The study coordinator will schedule the initial in-person intervention visit within two to three weeks after informed consent. Baseline assessments will be conducted by phone, online, or on paper forms within one week of initial visit. Informed consent, baseline assessments, and initial visit are also allowed to occur in this sequence on the same day, depending on patient's preference and provider availability.

The initial intervention visit will be followed by one-, two-, and three-month visits. All the study providers will complete a PC checklist after each study intervention visit in both models of care. Patients will complete ESAS, DT, and PHQ-9 before the intervention visit and other assessments after the study visits, respectively. ESAS, PHQ-9, and DT results will be given to hepatologist in Model 2 right before the study visit, to be used during the study visits. These three comprise the intervention toolkit for Model 2. Providing these data to PC providers (in Model 1) as a part of a study would represent a deviation of their routine clinical practice. Follow-up assessments will be conducted at 6, 9, and 12 months from the initial visit. No clinical intervention is mandated at these follow-up time points.

In the event of a patient undergoing LT, or admitted to home or inpatient hospice within three months from enrollment, the PC intervention will be stopped. However, data collection will continue as scheduled, for both patients and caregivers, until the 12-month follow-up. Crossover or contamination is minimized by providing contact cards to participating dyads, so they can alert their study coordinators or providers during any unscheduled inpatient admission.

Potential barriers to recruitment include. Patient factors - health related (such as uncertain prognosis/complications and physical and mental challenges) and social barriers (fear, resistance); Caregiver factors such as mistrust, limited information, beliefs, fear of intrusion, and false hopes.

Possible solutions. Involving patients and caregivers as stakeholders has helped develop a Recruitment and Retention strategy, the key elements of which include providing educational information about the project to caregivers and patients, simplified informed consent, most research visits integrated within routine clinical care for hepatologist-led group, availability of study coordinator making appointments for PC led group, patient remuneration, and continuous monitoring of enrollment targets from each site.

Retention. The most significant barrier to retention is the projected mortality rate and transplants among enrolled patients. Attrition rates will vary with the severity of disease, and $20 \%$ attrition has been considered in the statistical power calculations. Approximately $50 \%$ on the transplant list will actually get transplanted. However, by excluding patients with model for end-stage liver disease (MELD) >30 who are likely to be transplanted within three months, we expect only a small number to receive LT before the three-month primary end point.

\section{Data Collection}

All questionnaires are self-administered and can be completed on paper or online or by phone, as outlined in Table 4. 
Table 4. Main Study Measures and Data Collection Protocol

\begin{tabular}{|c|c|c|c|c|c|c|c|}
\hline & Baseline & 1 Month & 2 Months & 3 Months & 6 Months & 9 Months & 12 Months \\
\hline $\begin{array}{l}\text { Liver disease specific QOL } \\
\text { (FACT-Hep) }\end{array}$ & $X$ & $X$ & $X$ & $X$ & $X$ & $X$ & $X$ \\
\hline Overall QOL (PROMIS-29) & $X$ & & & $\mathrm{X}$ & $X$ & $X$ & $\mathrm{X}$ \\
\hline Symptom assessment (ESAS) & $\mathrm{X}$ & $\mathrm{X}$ & $\mathrm{X}$ & $\mathrm{X}$ & $\mathrm{X}$ & $\mathrm{X}$ & $\mathrm{X}$ \\
\hline Depression severity (PHQ-9) & $\mathrm{X}$ & $\mathrm{X}$ & $\mathrm{X}$ & $\mathrm{X}$ & $\mathrm{X}$ & $\mathrm{X}$ & $\mathrm{X}$ \\
\hline DT & $\mathrm{X}$ & $\mathrm{X}$ & $\mathrm{X}$ & $\mathrm{X}$ & $\mathrm{X}$ & $\mathrm{X}$ & $\mathrm{X}$ \\
\hline $\begin{array}{l}\text { Satisfaction with care } \\
\text { (FAMCARE-P 13) }\end{array}$ & $X$ & $\mathrm{X}$ & & $X$ & $X$ & $X$ & $X$ \\
\hline $\begin{array}{l}\text { Goal concordant care } \\
\text { questionnaire } \\
\text { (patients and caregivers) }\end{array}$ & $X$ & & & $X$ & $X$ & $X$ & $\mathrm{X}$ \\
\hline $\begin{array}{l}\text { Caregiver burden (Zarit } \\
\text { Burden-12) }\end{array}$ & $X$ & $X$ & & $X$ & $X$ & $X$ & $X$ \\
\hline $\begin{array}{l}\text { Qualitative interviews } \\
\text { (patients and caregivers) }\end{array}$ & & & & $\begin{array}{l}X \text { (after three months } \\
\text { of intervention phase) }\end{array}$ & & & \\
\hline
\end{tabular}

DT, distress thermometer; FACT-Hep, functional assessment of cancer therapy-hepatobiliary; FAMCARE-P, Family Satisfaction with Cancer Care-Patient; PROMIS-29, Patient Reported Outcomes Measurement Information System; QOL, quality of life.

\section{Qualitative methods}

We aim to conduct up to 98 patient-caregiver dyad interviews, all providers who underwent the PC training for hepatologists, and PC providers who underwent the study algorithm. Years of experience among providers will be ascertained. All interviews will be audio recorded, with permission under a separate consent. These will be transcribed verbatim. Grounded theory will be used to analyze these interviews, and emerging themes will be coded. ${ }^{34}$ Interviews will be transcribed, and NVIVO 10 will be used for qualitative data analysis.

\section{Statistical analysis plan}

Sample size and power. Published estimates show that the average FACT-Hep total score is 143.0 points (standard deviation [SD] 20.6), and a difference of 9 points reflects a minimal clinically important difference (MCID). ${ }^{35}$ We plan to enroll 90 patients in each of the 14 centers, resulting in 1260 enrolled patients (630 in each trial arm). Conservatively, accounting for $20 \%$ attrition we plan for 1008 analyzable patients. The study has $>90 \%$ power to detect a MCID in the primary outcome between the two randomized arms, for a range of SDs and intraclass correlation coefficients reflecting correlation of outcomes within a cluster (site).

Analysis for the primary hypothesis. All major treatment comparisons between the randomized groups in this trial will be performed according to the principle of "intention-to-treat," that is, subjects will be analyzed (and outcomes attributed) according to the treatment group to which patients were randomized. However, patients who undergo transplantation or hospice transfer before the threemonth end point will not be included in the primary analysis. Statistical comparisons will be performed using two-sided significance tests. To account for the cluster-based randomization we will utilize a linear mixed model with treatment arm indicator (binary variable) as a fixed effect and site as the random effect. ${ }^{36}$ The model for the primary outcome will consider the treatment binary variable, as well as baseline
QOL and randomization strata. Rejection of the null hypothesis stating that coefficient for the treatment variable is zero will provide evidence for presence of the treatment effect. Analyses will be performed with SAS version 9.4 and R version 3.4.2 statistical software.

In addition, we will assess heterogeneity of treatment effects across two prespecified subgroupings: (1) liver transplant eligible versus ineligible and (2) liver disease severity as assessed by MELD score above and below a threshold of 20. We will assess a potential differential treatment effect across subgroups by utilizing the linear mixed regression model with main effects and interaction between the randomized treatment variable and each of the two prespecified subgrouping variables. In addition to the formal assessment of interactions, effects of the treatment strategy will be estimated (with $95 \%$ confidence interval) in each of the described above subgroups. These descriptive summaries will be interpreted in conjunction with the formal interaction tests.

\section{Study Implementation}

\section{Patient and key stakeholder engagement}

We developed a research advisory board (RAB) through nominations of members from each participating center. The RAB members are patients with liver disease and caregivers with varied experiences such as those on the liver transplant list, those successfully transplanted in the past, and those who were removed from the transplant list. The RAB mission is to ensure that all aspects of the study are patient/caregiver focused. The RAB collaborated throughout the planning phase and will continue to provide input through the conduct/implementation and dissemination phase. Our meaningful engagement is maintained through regular board calls and journal club discussions. RAB members are represented in the executive committee as well.

\section{Data safety monitoring board}

A data safety monitoring board (DSMB) is formed to monitor patient safety and to review performance of the 
study. This independent board includes a Chair with both clinical and research expertise, senior statistician with prior DSMB experience, and additional clinicians with expertise in the clinical area. DSMB will convene every six months to review the accumulating data and make recommendations regarding continuation of the study.

\section{Fidelity monitoring}

To monitor consistent intervention delivery across diverse health care systems, we have developed intervention fidelity process. The process consists of required completion of a PC checklist by the providers in both models after each study visit and monitoring of the scores at the end of three-month intervention with expectation that $90 \%$ of the maximal score has been achieved. Clinical notes are entered for each intervention visit as a part of standard medical care. Periodically, selected clinical notes at each site will be reviewed and compared to the PC checklist to validate the accuracy of the latter. If there is lack of congruency or incomplete intervention, the hepatologists will be alerted and given additional training, if needed.

Dose of intervention will be equivalent between the two comparative models, as defined by the study visits. Both groups can have any additional visit(s) as required clinically.

Study contamination is avoided by the cluster-based randomization scheme, whereby all providers follow the same procedure across patients.

\section{Limitations}

Given the multifaceted intervention, it may remain unclear which components of PC are absolutely essential. However, this is the nature of PC, and our checklist will discern the details. Further research will be continued to identify the key elements, which led to improvement in outcomes.

If the primary hypothesis fails, it will be an important contribution to the field regarding models of PC delivery. The effect estimates and corresponding confidence intervals will inform future interventions and clinical practice.

Given the nature of ESLD leading to acute decompensation events and unpredicted/unscheduled hospitalizations, potential for contamination exists, especially in the hepatologist-led model. Our study procedures to alert the study coordinator will minimize this, and if this occurs, it will be captured in the research database.

Fidelity monitoring will rely on documentation only. Audio recordings of study visits were considered but due to strict rules on protecting confidentiality of patients and providers at different health care systems, they were not included.

\section{Dissemination}

Our network of hepatology providers within VA and nonVA sites will provide an opportunity to widely disseminate the findings throughout organizations such as the American Association for the Study of Liver Disease, the leading entity in the field of hepatology. In addition, we plan to disseminate the study findings to patient and caregiver community through social media and patient friendly newsletters. The study results are projected to inform and change the clinical practice guidelines.
The demonstration of success of PCA-Hep and superiority in our study will provide high quality evidence justifying inclusion of PC training into the standard curriculum for subspecialty training in hepatology. Furthermore, the additional time spent by the hepatologist on discussion of matters such as advance care planning would support a higher level of billing and make the model self-sustainable.

\section{Acknowledgments}

This work is supported by a Patient-Centered Outcomes Research Institute (PCORI) Award (PLC-1609-36714). The statements in this article are solely the responsibility of the authors and do not necessarily represent the views of the Patient-Centered Outcomes Research Institute (PCORI), its Board of Governors, or Methodology Committee.

The trial is registered at clinicaltrials.gov identifier NCT03540771.

This is a study protocol and thus does not contain or reflect any primary data.

\section{Author Disclosure Statement}

The authors report no conflicts of interest.

\section{References}

1. Udompap P, Kim D, Kim WR: Current and future burden of chronic nonmalignant liver disease. Clin Gastroenterol Hepatol 2015;13:2031-2041.

2. Tapper EB, Parikh ND: Mortality due to cirrhosis and liver cancer in the United States, 1999-2016: Observational study. BMJ 2018;362:k2817.

3. Peng JK, Hepgul N, Higginson IJ, et al.: Symptom prevalence of patients with end-stage liver disease: A systematic review. BMJ Support Palliat Care 2016;6:401-402.

4. Bajaj JS, Reddy KR, Tandon P, et al.: The 3-month readmission rate remains unacceptably high in a large North American cohort of patients with cirrhosis. Hepatology 2016;64:200-208.

5. Scaglione SJ, Metcalfe L, Kliethermes S, et al.: Early Hospital Readmissions and Mortality in Patients with Decompensated Cirrhosis Enrolled in a Large National Health Insurance Administrative Database. J Clin Gastroenterol 2017;51:839-844.

6. Bajaj JS, Wade JB, Gibson DP, et al.: The multidimensional burden of cirrhosis and hepatic encephalopathy on patients and caregivers. Am J Gastroenterol 2011; 106:1646-1653.

7. Mazzarelli C, Prentice WM, Heneghan MA, et al.: Palliative care in end-stage liver disease: Time to do better? Liver Transpl 2018;24:961-968.

8. Poonja Z, Brisebois A, van Zanten SV, et al.: Patients with cirrhosis and denied liver transplants rarely receive adequate palliative care or appropriate management. Clin Gastroenterol Hepatol 2014;12:692-698.

9. Langberg KM, Taddei TH: Balancing quality with quantity: The role of palliative care in managing decompensated cirrhosis. Hepatology 2016;64:1014-1016.

10. World Health Organization: WHO Definition of Palliative Care. www.who.int/cancer/palliative/definition/en (last accessed January 2, 2018).

11. Rabow M, Kvale E, Barbour L, et al.: Moving upstream: A review of the evidence of the impact of outpatient palliative care. J Palliat Med 2013;16:1540-1549. 
12. May P, Normand C: Analyzing the impact of palliative care interventions on cost of hospitalization: Practical guidance for choice of dependent variable. J Pain Sympt Manag 2016;52:100-106.

13. Zimmermann C, Riechelmann R, Krzyzanowska M, et al.: Effectiveness of specialized palliative care: A systematic review. JAMA 2008;299:1698-1709.

14. Kathpalia P, Smith A, Lai JC: Underutilization of palliative care services in the liver transplant population. World $\mathrm{J}$ Transpl 2016;6:594.

15. Zimmermann C, Swami N, Krzyzanowska M, et al.: Perceptions of palliative care among patients with advanced cancer and their caregivers. CMAJ 2016;188:E217-E227.

16. Fricker Z, Serper M: Current knowledge, barriers to implementation, and future directions in palliative care for end stage liver disease. Liver Transpl 2019;25:787-796.

17. Verma M, Navarro V: Patient-centered care: A new paradigm for chronic liver disease. Hepatology 2015;62:988990.

18. Wittenberg-Lyles E, Goldsmith J, Ferrell B, et al.: Assessment of an interprofessional online curriculum for palliative care communication training. J Palliat Med 2014; 17:400-406.

19. Kavita V, Dharmarajan MD: Primary palliative care education in specialty oncology training more work is needed. JAMA Oncol 2016;2:858-859.

20. Kelly SG, Rice JP: Palliative care for patients with endstage liver disease: The role of the liver team. Clin Liver Dis 2015;6:22-23.

21. ASCO Guidelines: Clinical Tools and Resources. https:// www.asco.org/sites/www.asco.org/files/pc_pco_checklist_ 1.18.12.pdf. (Last accessed December 3, 2016).

22. National Consensus Project for Quality Palliative Care. Clinical practice guidelines for quality palliative care. Kansas Nurse 2004;79:16.

23. Pizzo PA, Walker DM, Bomba PA: Dying in America: Improving Quality and Honoring Individual Preferences Near the End of Life. Washington, DC: Institute of Medicine, 2014.

24. Back AL, Arnold RM, Baile WF, et al.: Faculty development to change the paradigm of communication skills teaching in oncology. J Clin Oncol 2009;27:1137-1141.

25. Baile WF, Buckman R, Lenzi R, et al.: SPIKES - a six-step protocol for delivering bad news: Application to the patient with cancer. Oncologist 2000;5:302-311.
26. Breitbart WH, Chochinov H, Passik SD.: Psychiatric aspects of palliative care. In: Doyle D, Hanks GW, MacDonald N (eds): Oxford Textbook of Palliative Medicine, 2nd ed. New York: Oxford University Press, 1998, pp. 933 959.

27. NCCN Clinical Practice Guidelines in Oncology: Distress Management (Version 1 2014). http://williams.medicine.wisc .edu/distress.pdf. 2014. (Last accessed December 3, 2016).

28. Holland JC, Andersen B, Breitbart WS, et al.: Distress management. J Natl Compr Cancer Netw 2013;11:190-209.

29. Childers JW, Back AL, Tulsky JA, et al.: REMAP: A framework for goals of care conversations. J Oncol Pract 2017;13:e844-e850.

30. Schell JO, Arnold RM: NephroTalk: Communication tools to enhance patient-centered care. Semin Dialysis 2012;25: 611-616.

31. Five steps to advance care planning (Endlink, Northwestern University): http://endoflife.northwestern.edu/advance_care_ planning/steps.pdf. (Last accessed June 3, 2017).

32. Puchalski C, Ferrell B, Virani R, et al.: Improving the quality of spiritual care as a dimension of palliative care: The report of the Consensus Conference. J Palliat Med 2009; 12:885-904.

33. National Coalition for Cancer Survivorship: Cancer Survivor Toolbox. www.canceradvocacy.org/resources/cancersurvival-toolbox/special-topics/living-beyond-cancer. (Last accessed June 3, 2016).

34. Glaser BG, Strauss AL: The Discovery of Grounded Theory: Strategies for Qualitative Research. Routledge, 2017.

35. Steel JL, Eton DT, Cella D, et al.: Clinically meaningful changes in health-related quality of life in patients diagnosed with hepatobiliary carcinoma. Ann Oncol 2006;17: 304-312.

36. Verbeke G, Molenberghs, G.: Linear Mixed Models for Longitudinal Data. New York: Springer-Verlag, 2000.

Address correspondence to:

Manisha Verma, MD, MPH

Department of Digestive Diseases and Transplantation

Einstein Healthcare Network 5501 Old York Road Philadelphia, PA 19141

E-mail: vermam@einstein.edu 